\title{
How to Do Knowledge Module Finishing
}

\author{
Shunpeng ZOU ${ }^{1}$ and Xiaohui ZOU ${ }^{[0000-0002-5577-8245], 2}$ Xiaoqun Wang ${ }^{2}$ \\ ${ }^{1}$ China University of Geosciences (Beijing) 29 Xueyuan Road, 100083, China \\ 2 Peking University Teacher Teaching Development Center, Room 415,Beijing, 100871 China \\ 949309225@qq.com and zouxiaohui@pku.org.cn,xqwang@pku.edu.cn
}

\begin{abstract}
The aim is to introduce a simple and unique tool to realize the ergonomics of man-machine collaborative research on "governance of the country". This method: First, the original text is imported, and then, a Chinese character board is generated, and then the language points, knowledge points, and original points are matched. The feature is human-machine collaboration. The result is that the Chinese character is tessellated, the Chinese chess spectrum is made, and the original chess is played. The knowledge module can be used for finishing. Its significance is: the language board, the knowledge game, and the original board game are digital, structured, and formal indirect ways to achieve automatic calculation: language points, knowledge points, and original points. The five menu-based knowledge modules of this example can be layered and distributed, and at least sixteen knowledge modules can be finished. Man-machine can be repeated calls. This is a contribution to the interpretation or understanding of texts.
\end{abstract}

Keywords: Linguistic cognition, Brain-Machine Integration, Indirect formalization, Computer-aided Teaching, Knowledge Module Finishing

\section{Introduction}

Through research, we have found that due to language understanding, knowledge expression, and habits of people's thinking, etc., what people desperately need is not only interpersonal academic communication but also the intelligent ability of human-computer interaction, especially in this area of ideology and politics education informatization. This paper takes computer-assisted study of the essence of Xi Jinping's thoughts on ruling and managing the country as an example. It attempts to explain how man-machine combination can be used as a new method of knowledge module finishing to help understand the basic principles and core strategies. For example, all of them are interpreting Xi Jinping's thoughts on ruling the state, but there are many versions of interpretations by experts. Especially in the social environment where computers and smart phones and other types of artificial intelligence terminals are popular today, they are more prone to the appearance of similar blind people's images. This is not conducive to the unity of one mind, one knowledge and action, and then concentrates on working together to make the nation a good game. Regardless of the bottom-up or the top-down, the key lies in: How to achieve the goal of swiftly realizing the nation's ups and downs and steadily realizing the goal of ruling the country? Computer-assisted research on Xi Jinping's idea of governing the country is helpful for us to give full play to the comprehensive advantages of human-computer-brain collaborative processing of information.

Therefore, the purpose of this paper is to use a simple and unique method and tool to collaboratively study the essence of $\mathrm{Xi}$ Jinping's thoughts on governing the country, which can be summed up briefly as a 
computer-aided study of the essence of ruling the country, which is characterized by a reasonable division of labor complementary advantages.

\section{$2 \quad$ Method}

\subsection{The scientific principles on which this method is based}

The method adopts the indirect formalization method and the indirect calculation model. The principle of logic by order and position, the principle of bilingual mathematics and the principle of generalized translation, which are the three basic laws of the digital and textual chessboard, are the scientific support. Among them, the order and position of the numbers and the order and position of the words combine to form the double-chess board principle. It explains the specific order and position of all the structural units of Chinese on the board. This determines the most basic sequencing positioning development environment and calling platform for human-computer interaction, and any piece of text, any sentence, any phrase or even any word must be just a series of choices. It is not only theoretically applicable to the intelligent analysis of any text, but also in practice, through a series of targeted large and small double chessboards, and the corresponding knowledge module for the specific text. Because it is limited to plain text analysis, its scalability reaches its limit; because it is limited to Chinese examples, it can reach its limits. In other words, any discourse fragment or text, which contains language points, knowledge points and original points (referred to as "three points") and even featured software modules, such as: knowledge maps and mind maps, can be recorded from the menu of the "three points" come from the combination and construction. Since the task of this article is limited to demonstrating the method with its characteristics through a typical example, the explanation is not extended.

\subsection{Taking the formal study of the ideology of "Ruling the country" as an example}

Taking the formal study of the ideology of "Ruling the country" as an example, demonstrating new methods and tools.

One of the authors took the case of "big data processing and knowledge management based on doublechessboard: taking abstracts of papers and patents as an example" and "taking an abstract of the organizational profile and personal profile in the thinking and exploration of various high-end think tanks as an example" In practice, we promote the popularization of big data processing and knowledge management based on doublechessboards. Such new methods and tools for human-computer interaction have made great progress. Therefore, the author here introduces the Marxist Academic Forum and tries to make a set of typical examples in the study of Xi Jinping's themes, main lines, and scientific theoretical systems. [1] [2] Its popularization involves the application of a series of "diversity" models. Its empirical analysis has proved that macroscopic models and microscopic models can be used not only for the "involved research object" of the "involved system" discipline. The four theoretical questions, interlocking disciplines, conceptual systems, and methodological systems, give clear explanations, and then can use indirect formalization and indirect calculations of system engineering solutions to deal with terminological descriptions at all levels. The previous examples of the author reveal that the research object of the (community) teaching management formed by the "diploid" model of university education and management is "the discussion of advanced talent training and advanced knowledge", and the nature of the discipline is "the overlapping of teaching and management". "The concept system and method system relate to a series of activities "classification" and "cases" formed by the "teaching" and "management" of the campus classroom. Among them, the rough concept can get the basic concepts involved in macro models. The framework can then be further subdivided to obtain the basic terms involved in the microscopic model. [3] 
This will further systematize Xi Jinping's idea of ruling the state, so that further formation of a universal scientific theory system through computer-aided research is helpful. For the time being, it is not necessary to construct an expert knowledge system for human-computer interaction that requires a human-computer interaction native language environment that is compatible with personalization and standardization. Only a set of specific and operable embodiments is cut in.

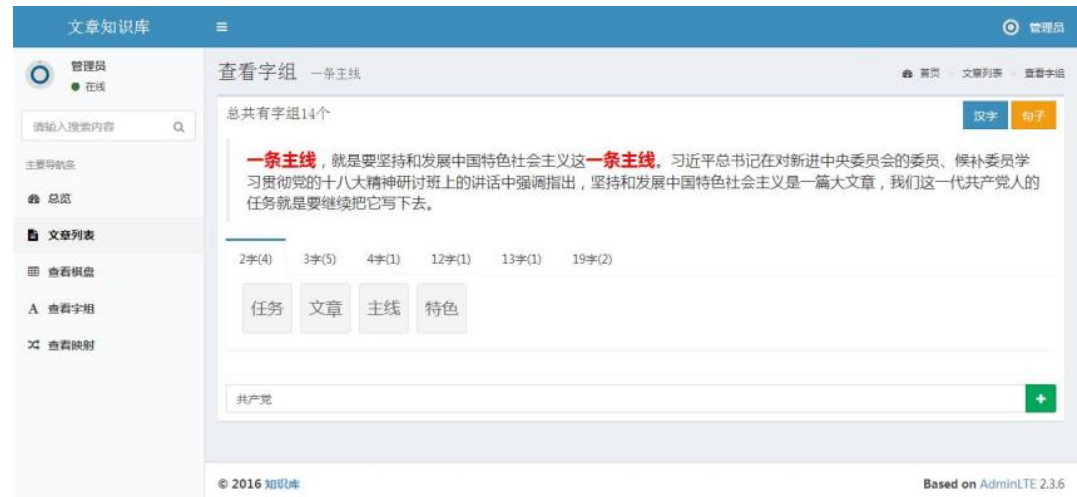

Figure 1 "One main line" example of knowledge module diagram

As shown in Figure 1, one prominent line is to uphold and develop the main line of socialism with Chinese characteristics. Below the speech fragment there is a series of digits that record the selected word group (there are several) of the user, visible below, and formatted and highlighted the "task, article, main line, feature". These are formalized, digitized, and formatted textual texts, that is, the ideological and political education information methods described in this article. They are to achieve this step by step:

The first step is to enter the "Add Article" interface we provide（http://kb2.sloud.cn/article/add）

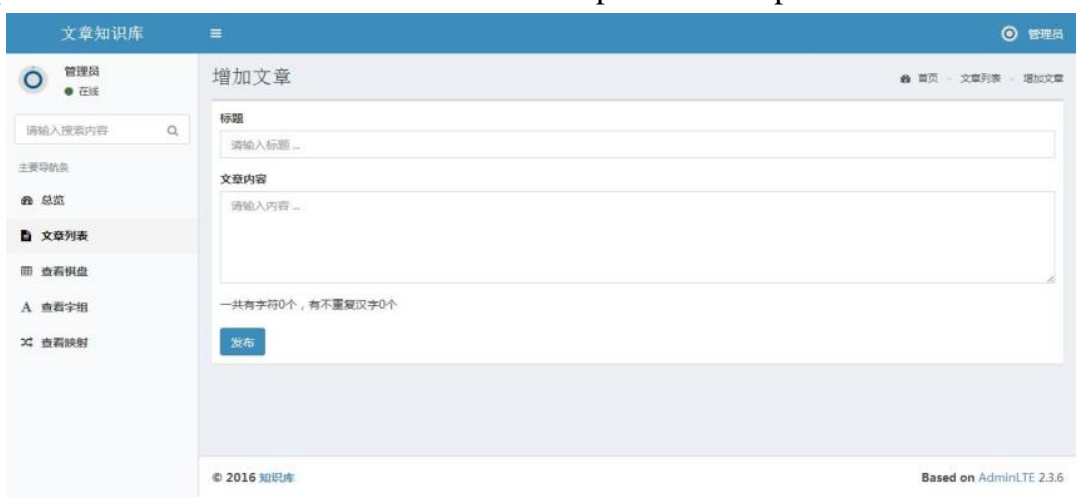

Figure $1 \mathrm{a}$ The window for adding articles is the man-machine interface

The second step, fill in the "Article Content" and "Title" (see the following example)

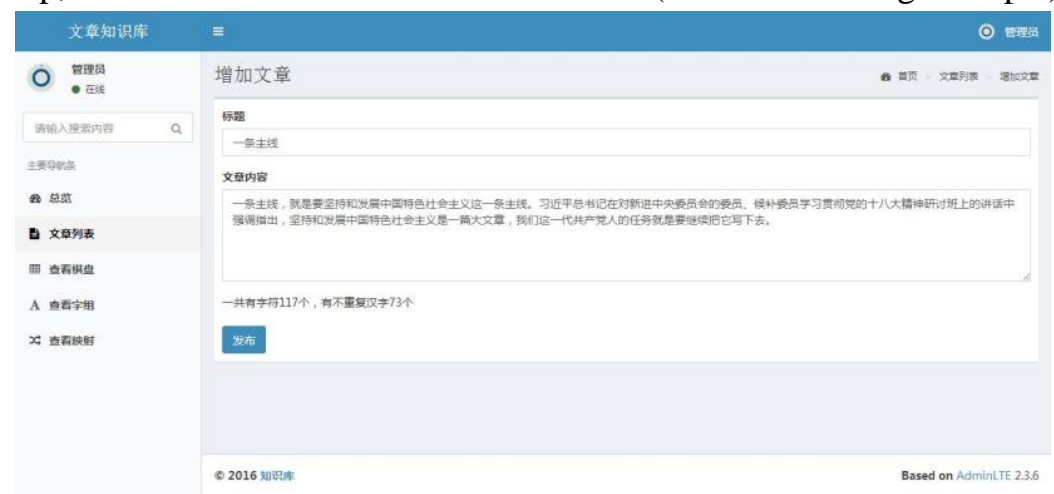

Figure $1 \mathrm{~b}$ Fill in or import the text of "Title" and "Article Content" in the window of the increase article

The third step, release (visible) 


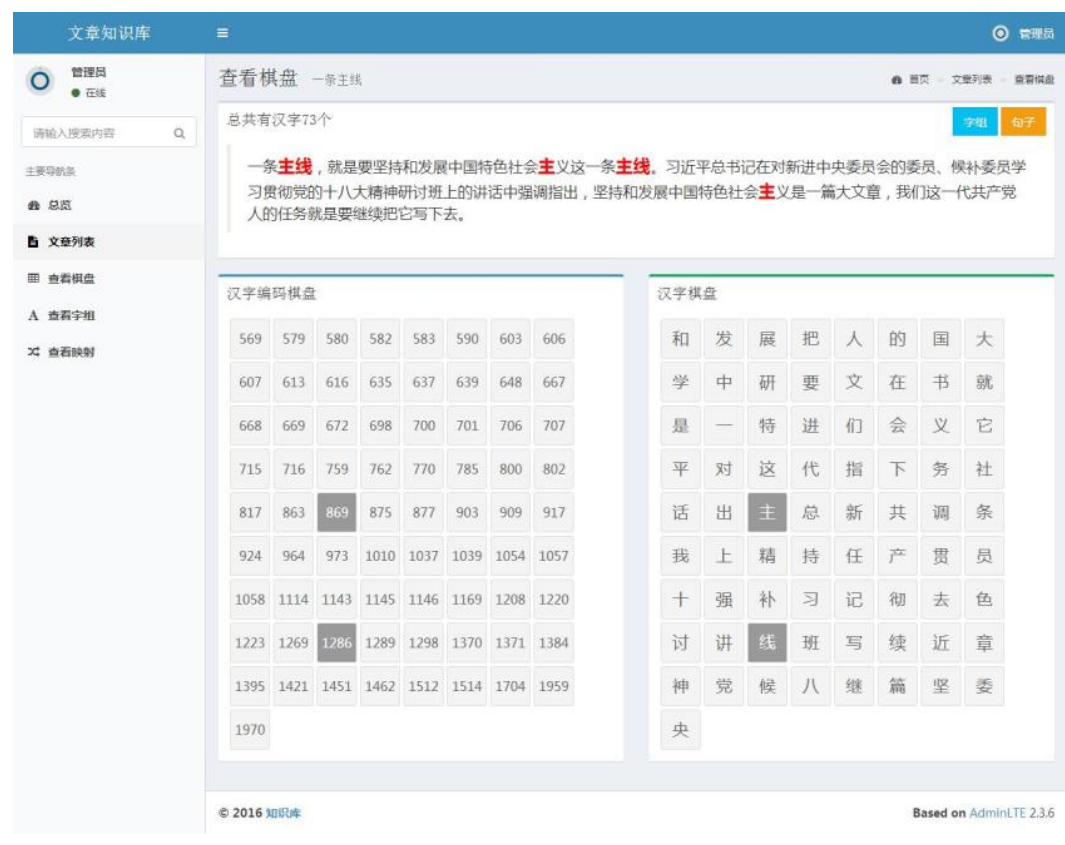

Figure $1 \mathrm{c}$ Check the board

As shown in Figure 1and Figure 1a, the first step, the second step and the third step are all extremely silly and easy to understand (the pupils can also quickly understand and learn how to operate). From Figure 1b, after the text is imported, the system will automatically count the total number of characters and their non-repeated number of words (in this case, the non-repetitive Chinese characters, that is, the smallest language structure form unit of Chinese or Chinese) is the most basic language point. From Figure 1c, the language points and knowledge points implied in the text can be highlighted by means of selection and clicking on the Chinese character board. For example, the "mainline" is where the matching of language points and knowledge points or even original points overlaps. Another example is that "ism" is very interesting. At this point, the language points and knowledge points and even the original points are only similarly matched, similar, or similar. What's more interesting is that each person's choice or thinking can be different, but they must be limited to the range of the double word board; the machine can also have its own algorithm or choice. The most interesting thing is that not only man-machines can have their own choices, but also, the combination of man-machine formation and the dual-brain collaborative selection, that is, intelligence, is better than pure human brain intelligence and purely computer-based intelligence in both physics and practice. Smart has more advantages. The unique advantages of the discovery and invention of the double-word board are specifically described in another book. With such knowledge module finishing methods and tools, we can better discuss the differences and links between their respective thinking, understanding and understanding. For example, we can better understand "a main line."

We can do an in-depth analysis and discussion on that sentence itself, and we can continue to analyze and think in depth on the basis of the intensive processing of basic knowledge modules. 


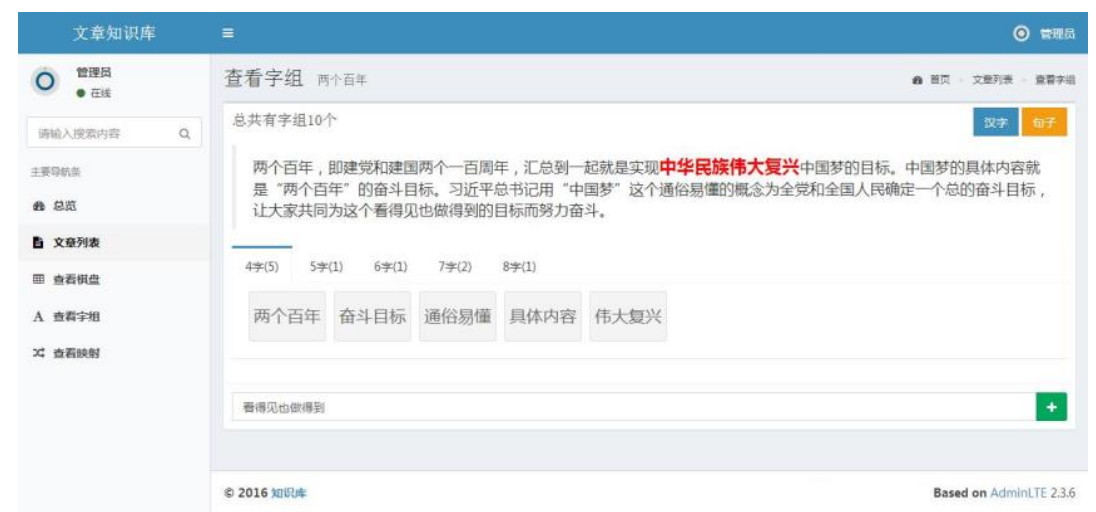

Figure 2 "Two hundred years" example of knowledge module diagram

As shown in Figure 2, the essence of two centuries is highlighted or expressed or understood even more through a series of word combinations that are related, similar or similar to each other. The most crucial is the human-computer interaction process. In terms of individuals and groups, human-computer interaction can be easily achieved, and even human-computer interaction and interpersonal networking can be achieved. The combination of such intellectual activities and artificial intelligence is far more than simply reading texts, even graffiti discussions on paper or chalkboard, and is more flexible and easier to retain, share, and have standard measurability. Readers can compare themselves（http://kb2.sloud.cn/article/219/group）。

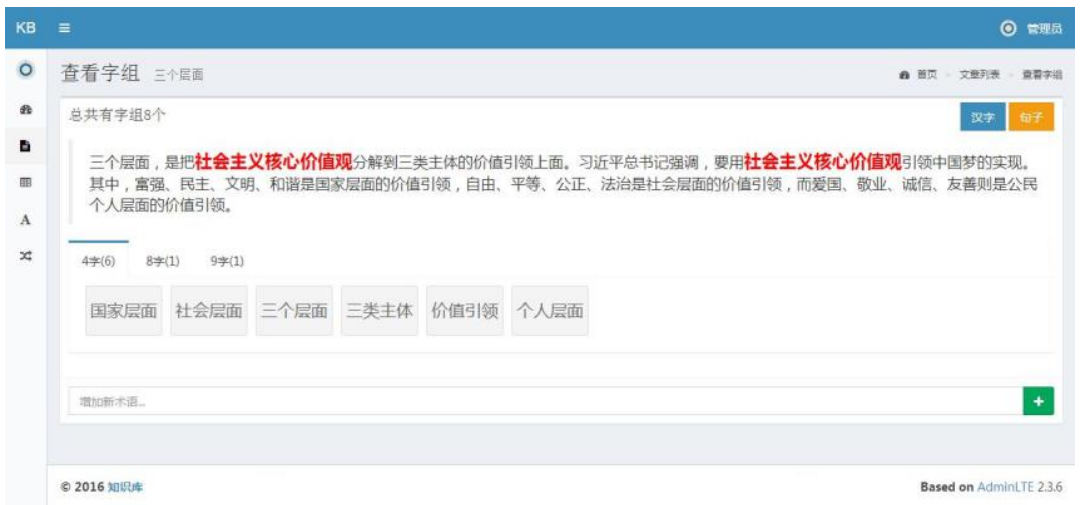

Figure 3 shows an example of a three-level knowledge module

As shown in Figure 3, in addition to the features illustrated in Figures 1 and 2 above, the author also emphasizes: "Wealth, Democracy, Civilization, Harmony," and "Freedom, Equality, Justice, the Rule of Law," and "Patriotic and Professionalism." Each of the three sequences of honesty and friendliness can be grouped together into three categories for an overall explanation and a corresponding knowledge module. Each keyword can be further explained individually and individually. And make more specific knowledge modules. This section is left to the reader to do it (http://kb2.sloud.cn/article/add) and make a corresponding comparison.

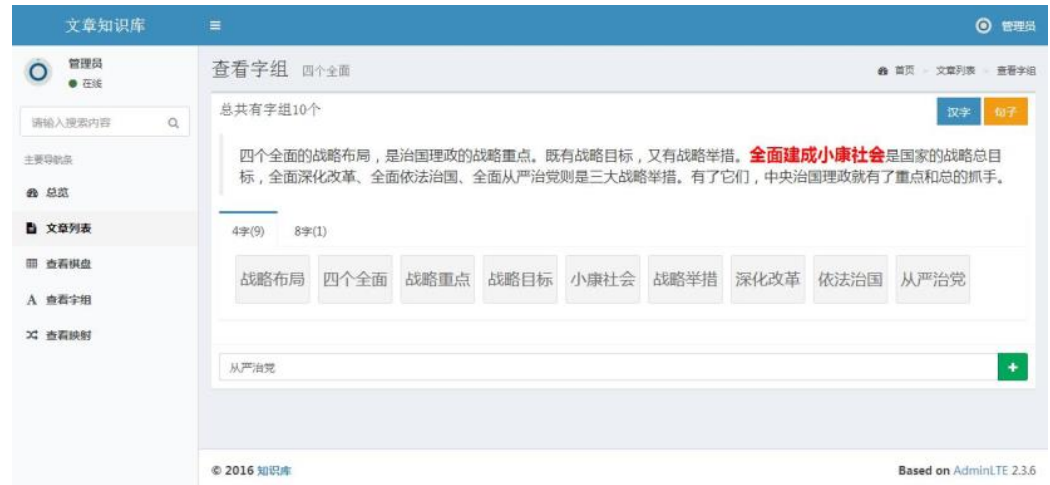


Figure 4: An example of a "four comprehensive" knowledge module

As shown in Figure 4, each of the four comprehensive aspects is not only an organic whole, but also has rich content in every aspect. Therefore, not only can it be made of four separate items, it is conducive to further study and understanding. The knowledge module, but also can take advantage of the situation, the content of each aspect is a system of combing, making a series of knowledge modules.

This is more meaningful than simply staying in these words. Ask the reader to try and compare.

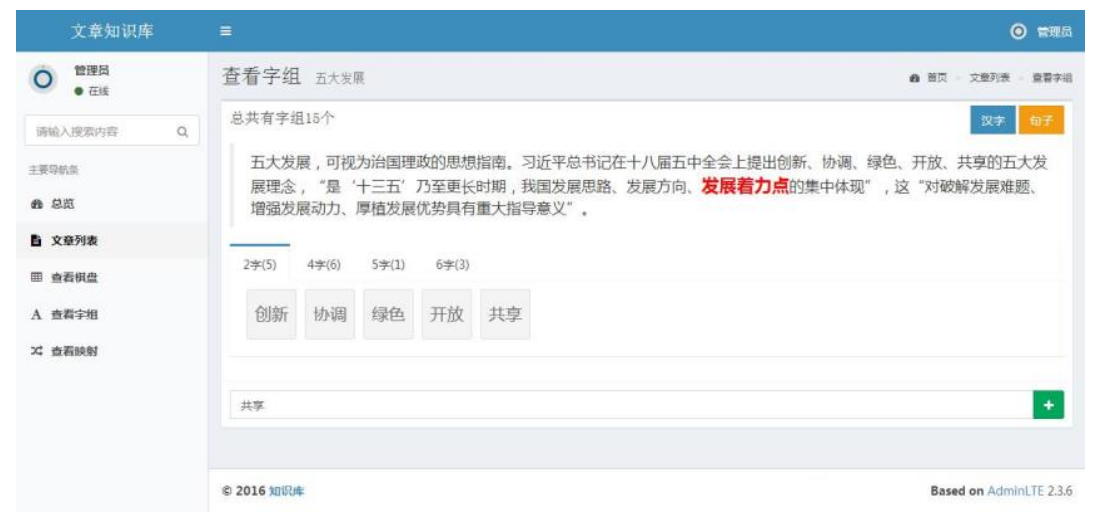

Figure 5 shows an example of a "five developments" knowledge module

As shown in Figure 5, this is just a screenshot. Readers can further review the knowledge modules (http://kb2.sloud.cn/article/222/group) in many aspects and even in combination with the aforementioned suggestions.

The above five aspects are interlocking. The main line for the development of socialism with Chinese characteristics must further clarify its direction, that is, realize the Chinese dream. With this goal, it is necessary to further clarify the value orientation of achieving the goal, that is, the core values of socialism; with value guidance, we must further clarify the current The strategic focus in the future and in a period of time will be four comprehensive strategic layouts; to achieve this strategic layout, the five major development concepts will be fulfilled. [5]

\subsection{Expert knowledge of human-computer collaboration System platform}

Expert knowledge of human-computer collaboration System engineering development platform: Creating a Chinese development environment compatible with personalization and standardization.

The author's further simplification and combing can be referred to as the ideological essentials of the "Governing the State and Managing the State" (enriched version).

Contains 116 non-repeating characters after clarifying the need to develop the main line of socialism with characteristics, we must further clarify its direction. The goal of achieving the great rejuvenation of the Chinese nation in two centuries is to realize the Chinese dream, and it is necessary to realize it clearly. Value orientation guides the values of the three levels of the country, society, and individual, that is, the socialist core values. Further, we must also clarify the strategic priorities for the current and future period, that is, four comprehensive strategic layouts that cover the building of a well-to-do society. Strategic goals, comprehensive deepening of reforms, comprehensively governing the country according to law, and comprehensively rigorously governing the party's three strategic initiatives requires the realization of this strategic layout. In the end, we must not only rely on but also honor the five development concepts of "innovation, coordination, greenness, openness, and sharing". . [6]

It was introduced into the expert knowledge system engineering development tool platform for human-robot collaboration, and the big data processing and knowledge management based on the double-word 
chessboard [8] can be implemented by following the rules of "sequencing and positioning, synonymous juxtaposition and consent" [7]. Specific practices and explanations:

The import can instantly generate the summary-specific double-word board, which is characterized by a non-repetitive semi-structured Chinese chess board (digitized) and its mapped purely structured digital board (numbers) and the unstructured summary (data) All at a glance, so that party and government cadres, experts, scholars, and teachers and students can work together to further refine their knowledge, without having to use any other programming language development environment based on the US standard information exchange code. Based on the "two broad categories of formalization strategies," we have created a specific implementation tool for the dual formal approach: the generalized bilingual information processing platform:

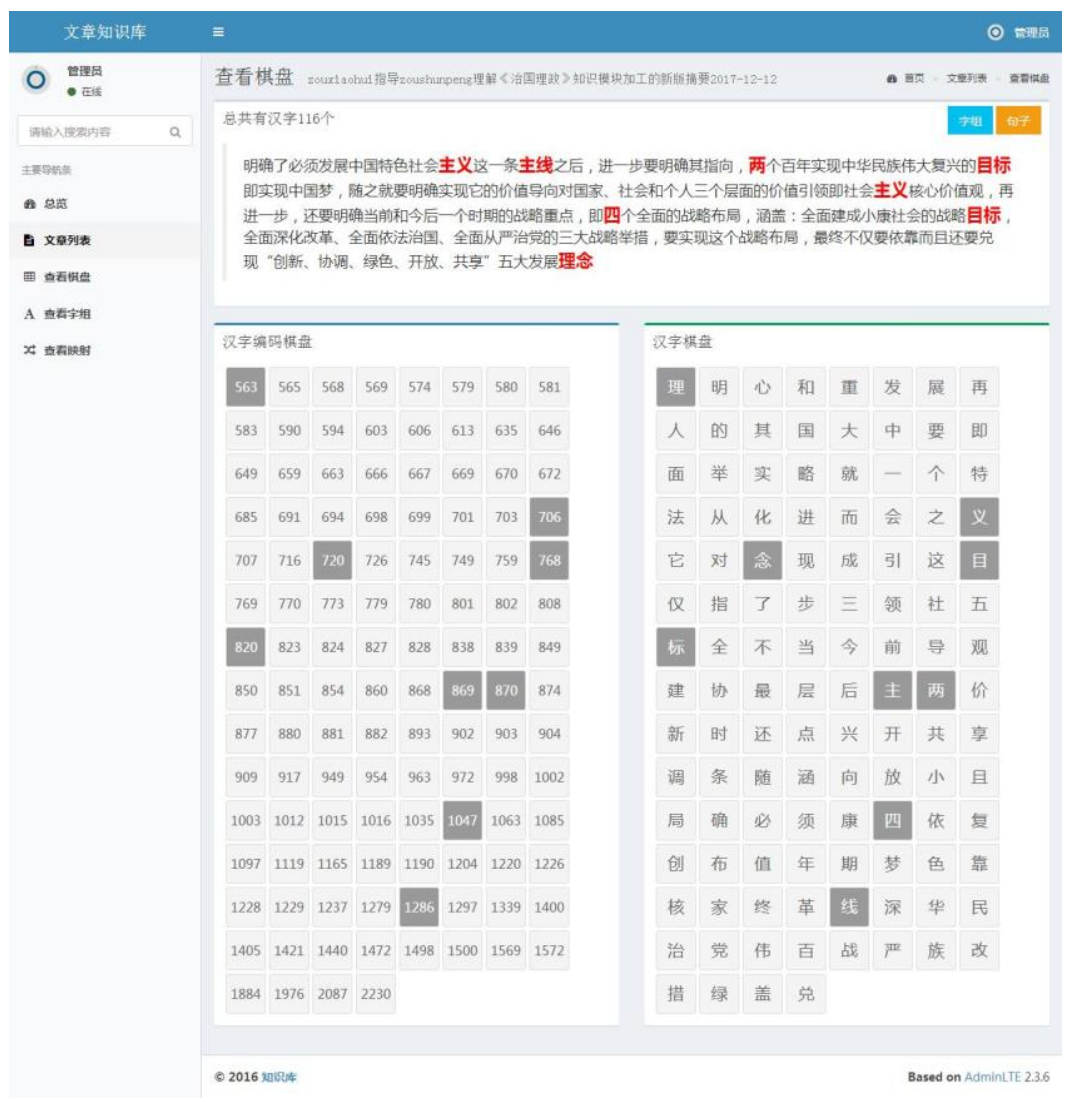

Fig. 6 Summary of the Chessboard Structure and Principle of the Analysis of the General Knowledge Module

As shown in Figure 6, this example is actually a sample version of the super silly programming development environment. It can not only automatically generate a double-word board, the unstructured abstract is the product of the human brain, that is, we further comb and refine on the basis of expert knowledge, which can not avoid the common disadvantages of the natural person.[9][10] By the computer by importing 226 characters and non-repeating Chinese characters 116 that is the summary, the summary automatically generates the abstract specific double-word board namely the semi-structured Chinese character board (matrix) and its mapped purely structured digital board ( Matrix) is usually difficult for natural people to do. This lays the foundation for human-computer collaboration and even man-machine collaboration - super silly programming development environment platform. Furthermore, we can use the large-scale chess game of "individual governance" as an example to carry out a large-scale cooperation of all parties, the whole army, and all walks of life in the country, and lay down a simplified version of the reference frame that can be considered comprehensively. . Among them, because the logical sequence order that the numerical chessboard and the Chinese character board follow is the same, there is a one-to-one correspondence between the two symmetrical 
matrices. Thus, the basis for the formal reasoning of the size premise is already in place. The rest is to achieve computer-aided research through the super-stupid programming development of the concrete double-word board. Through man-machine collaboration and interpersonal negotiation, the feature set implied by the abstract is extracted, and then the reference frame of reference is established.

The result is: the tessellation of the Chinese text and the chess genre of the Chinese text and the spirit of the ideological content, ensuring the essence of the abstract, ie the core idea and the style and characteristics of its expression, etc. Under the overall pattern, it is accurate and accurate.

The specific practices and steps are as follows:

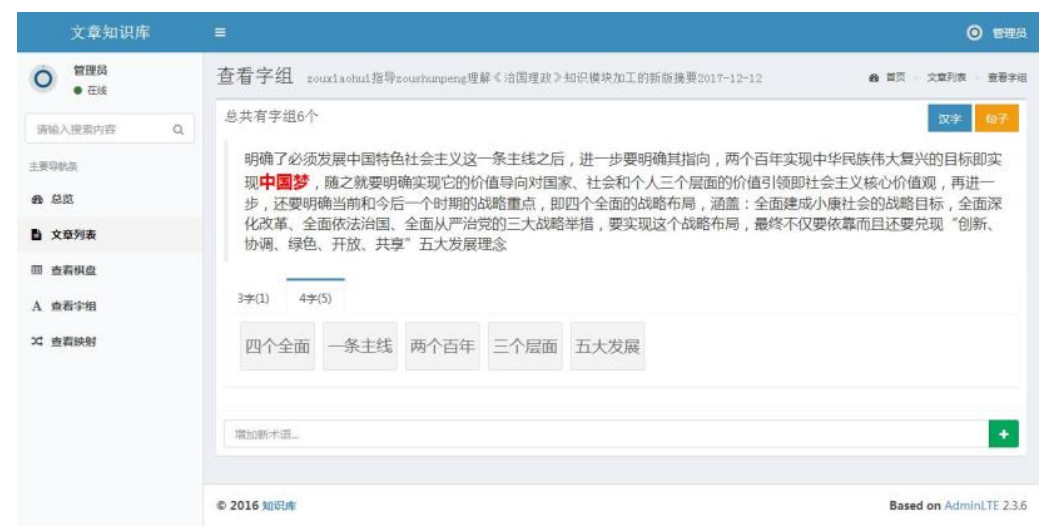

Figure 7 Summary of the General Knowledge Module in "Governing the State"

As shown in Figure 7, the "one main line, two hundred years, three levels, four comprehensive, and five major developments" in the abstract are identified by the user or the system, respectively, and then, through the "add new terms" approach, Structured text (summary) establishes a mapping relationship with structured text (the set of menus that match the language points and knowledge points in the box and the original points).

If the way that natural persons help computers do precise learning is to teach them how to choose a group of words, the reverse is that computer-assisted natural persons learn and study. Here, the user (natural person) finds a way to add a new term in the abstract, and lets the system (computer) react "a main line" (in the box) to establish a mapping relationship. Segmentation and tagging can be implemented on this hyper-silent programming development environment platform using only the native language (Chinese). In the same way, it can also be found in the abstract that "two hundred years, three levels, four comprehensive, and five major developments" are added one by one into the box and formalized. The main purpose is to tell the process of segmentation and labeling. The machine implements a computer-assisted set up of a series of mapping relationships.

Of course, we cannot demand that computer systems truly understand like natural persons: This "one line" is to continue to follow the path of socialism with Chinese characteristics. However, we can pass this keyword - whether it is a term or a common saying., Make a hyperlink to make it look like it really understands that "a main line" is to continue to follow the path of socialism with Chinese characteristics. Such a path or algorithm can all be told by the user and reused by the system.

\section{$3 \quad$ Result}

Simply put, this is how we can complete this kind of knowledge module finishing by human-robot collaboration. Attentive readers will remember and discover that we have said "respectively by the user or system to find out" such words. Its meaning is that it can be used as a way to help people, but it can also use machine-assisted methods, and can also use machine learning methods to collaboratively complete the matching 
of language points, knowledge points, and original points. This is the process of human-machine collaboration. The matching of terms and colloquialisms matches the language points, knowledge points, original points, and even matches with grammar, semantics, and pragmatics. Not only is it related to the double-word board that is composed of digits and words, but it is also linked to the "Chinese chess spectrum" consisting of bilingual Chinese and English. We regard terminology and colloquialism as alternative bilingualism. The three types of bilingual matching process are extensions of this study. In the process of man-machine collaboration, once the user (natural person) selected "one main line, two hundred years, three levels, four comprehensive, and five major developments" from the above abstract (unstructured), the computer system immediately They are all structured and formalized to lay the foundation for further automated invocation. In this process, the two parties are quite simple, but they are very useful. Embedding other methods (such as deep learning) will be more effective.

Although the above illustration is only a series of computer-assisted studies based on the ideological essentials of "Ruling the State," but it can at least prove that the combination of the human brain and the brain can indeed use super-stupid programming to process core knowledge modules. - This is the introduction of such texts and abstracts (by the natural person experts playing the role of the human brain) in the automatic generation of double-word board, but also to achieve human-computer interaction in a semi-automatic way to further generate the Chinese chess game - not only to throw a brick Moreover, experts from all walks of life, as well as teachers and students from all walks of life, can participate in the construction of a complete "governance of the state," and a system of scientific theories. In addition, computer-aided research and scientific theory systems can be conducted for various disciplines.

\section{Conclusion}

It can be seen that the human-computer interaction for knowledge learning, research, and large-scale production methods of processing based on the double- chessboard have a particularly important role and value for the large countries of higher education. This type of educational informatization approach, ie, the knowledge module finishing method used by the knowledge navigation device and the knowledge center behind it, is a systematic formalization method [11]. The practices of knowledge map [12] and knowledge graph [13] and mind maps [14] are not only compatible, but also complementary, as well as their "three points" and "three changes" to promote human-computer collaboration.

Its significance lies in the fact that the formalization Chinese characters as chess is automatically generated by the software, which can realize the automatic calculation of language point and knowledge point and the original point "three points". The specific performance of this paper is that only one or two or four and five can be used. Both the human brain and the computer can accurately reproduce the logical status of each point in the quintessence of the ideology of "governing the country" in the entire terminology system, fully embodying the dual innovations of character informatization and Chinese intelligence in sorting out texts and reading them.

\section{References}

1. Jiexia Xia, Qing Fang. Xi Jinping's Thought on Ruling the State and Managing State Affairs and the Latest Progress of Marxism in China[J]. Marxism Research, 2017, (01): 153- 155+2.

2. Zou Xiaohui, Zou Shunpeng. An Indirect Formalization Method Based on Bilingual Automatic Conversion [P]. Beijing: CN102253934A, 2011-11-23.

3. Zou Shunpeng. A New Study on Theories of College Education Discipline Construction [D]. China University of Geosciences (Beijing), 2012. 
4. Han Qingxiang. Comprehensively and Thoroughly Grasping Ten Important Aspects of Xi Jinping's Thought of Governing the Country and Managing the State [J]. Socialism with Chinese Characteristics, 2014,(06):24-28.

5. Han Zhenfeng. The Basic Content and Internal Logic of Xi Jinping's Thoughts on Governing the Country [N]. Guangming Daily, 2015-12-07(016).

6. Zou Xiaohui, Zou Shunpeng. Two Major Categories of Formal Strategies [J]. Computer Applications and Software, 2013, 30 (09): 194-199.

7. Zou, X.; Zou, S.; Ke, L. Fundamental Law of Information: Proved by Both Numbers and Characters in Conjugate Matrices. Proceedings 2017, 1, 60.

8. Zou Xiaohui. Double Chessboards [P]. Guangdong: CN104056450A, 2014-09-24.

9. Cai Juan. "Two Hundred Years" Struggle Goal Casts "Chinese Dream" [N]. Chinese Social Science Journal, 2013-05-27 (A05).

10. Cheng Chou. "Four Key Words" Interpretation of General Secretary Xi Jinping's Series Speech_— Interview with Professor Xin Ming of the Central Party School of the Communist Party of China [J]. Leader Wencui, 2014, (01): 7-22.

11. Zou Xiaohui; Zou Shunpeng. Bilingual Information Processing Methods and Principles. [J] Computer Applications and Software, 2015, 11

12. LIU Hongjing, TAN Liang. Research on Constructing Knowledge Map of SPOC Course Based on Teacher Perspective[J]. Electro-Educational Research, No.9, 2017

13. Qiao Kuo;Xiao Shijie. Application of Subject Knowledge Atlas in Constructing the Course Map of Middle School English[J].Modern Communication, 2017,18

14. He Jing. Analysis of the Role of Computer Mind Map Software in English Teaching [J]. Computer Fan, 2017, 10 